Ann. Biol. anim. Bioch. Biophys., I967, y (1), 29-32.

\title{
CESTROGÈNES URINAIRES CHEZ LA BREBIS GESTANTE OVARIECTOMISÉE
}

\author{
J. FÈVRE \\ avec la collaboration technique de Ginette LÉPINE \\ Station centrale de Recherches de Nutrition, \\ Centre national de Recherches zootechniques, 78-Jouy - en-Josas
}

L'étude de l'excrétion des œstrogènes chez la Brebis durant la gestation a montré que la voie urinaire était une voie mineure d'élimination, la voie fécale étant la voie principale. Cependant, dans la mesure où le pourcentage d'excrétion par 1'urine reste constant, l'élimination urinaire des œstrogènes peut être considérée comme un reflet de la production totale journalière par les organes sécréteurs.

$\mathrm{Si}$, en l'absence de gestation et en dehors de certains cas pathologiques tels que des tumeurs surrénaliennes, on sait que les ovaires sont la principale source d'œstrogènes chez les femelles des principales espèces, on peut se demander quelles sont; durant la gestation, les parts respectives des ovaires et de l'ensemble fœto-placentaire. C'est ce que nous avons cherché à connaître en procédant à des ovariectomies pendant la gestation, les ovaires chez la Brebis n'étant plus indispensables à partir du $5^{\circ}$ jour de gestation (Denamur et Martinet, I955).

\section{MATÉRIEL E'T MÉTHODES}

Deux brebis Ile-de-France gestantes furent ovariectomisées l'une $\left(n^{0} 3^{85}\right)$ le $99^{\mathrm{e}}$ jour de gestation, l'autre $\left(\mathrm{n}^{\circ} \mathrm{0} 9 \mathrm{3}\right)$ le $103^{\mathrm{e}}$ jour. La gestation s'est maintenue sans apport de progestérone exogène. A partir de l'opération et jusqu'à la mise bas, elles furent maintenues en cage à métabolisme. Les urines de 24 heures, séparées des fèces, furent recueillies tous les 2 ou 3 jours sauf pendant les 15 jours précédant la date présumée de mise bas, où elles furent recueillies quotidiennement.

Les méthodes utilisées furent les mêmes que celles qui ont été décrites dans un article précédent (FÈvRE et RoMbaUTS, I966), à savoir :

- étude quantitative : méthode de Brown adaptée au dosage dans l'urine de Brebis, suivie de la coloration de Kober-Ittrich;

- étude qualitative : spectres d'absorption, réactions colorées en milieu liquide, réactions colorées après chromatographie sur couche mince. 


\section{RÉSULTATS ETT DISCUSSION}

Les corps jaunes au moment de l'ovariectomie étaient fonctionnels :

Brebis 039 : 2 corps jaunes de poids moyen $597,2 \mathrm{mg}$ contenant $22 \mu \mathrm{g}$ de progestérone par gramme de tissu frais;

Brebis 835 : I corps jaune de 557,2 mg contenant $28 \mu \mathrm{g}$ de progestérone par gramme de tissu frais.

I a mise bas s'est produite normalement et les brebis ont donné naissance, 1'une (brebis 039) à deux agneaux vivants de $3800 \mathrm{~kg}$ et $3700 \mathrm{~kg}$, l'autre (brebis 835) à un agneau vivant de $4900 \mathrm{~kg}$.

Au point de vue qualitatif, les œstrogènes présents dans l'urine de Brebis gestante ovariectomisée sont les mêmes que dans le cas d'une brebis gestante intacte (FìvRE et RoMbaUTs, I966). La présence d'œstradiol I7 $\alpha$ semble bien établie de même que l'absence d'œtradiol $I_{7} \beta$. Les autres métabolites œestrogènes trouvés dans cette étude sont l'œstrone et un œstriol en faibles quantités, qui est soit l'estra I. 3. 5. (Io) triène $3, I 6 \alpha, I 7 \beta$, triol soit son I6 épimère.

Au point de vue quantitatif, l'œstradiol $I 7 \alpha$ est le métabolite principal. Nous n'avons pas, chez ces animaux ovariectomisés, retrouvé la même cinétique que celle qui avait été mise en évidence chez les animaux gestants normaux, mais les valeurs maximales sont en accord dans les différents cas, 60 à $80 \mu \mathrm{g}$ par 24 h pour l'œstra$\operatorname{diol} I_{7} \propto$ (fig. I).

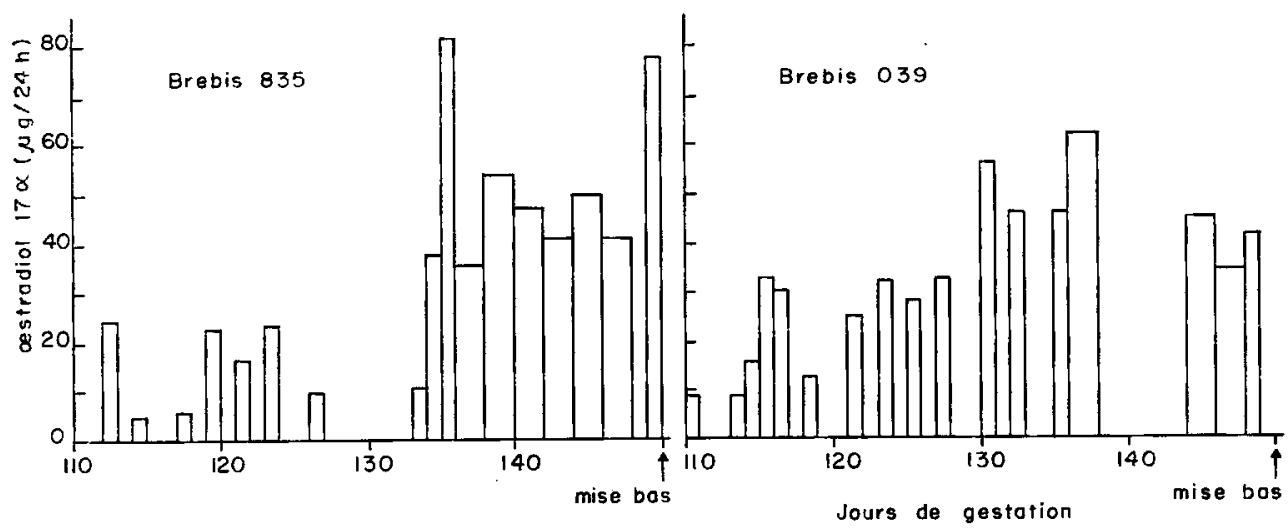

FIG. I. - Excrétion urinaire d'œstradiol $\mathrm{I} 7 \alpha$

Cependant, les quantités d'œstrone sécrétées par l'urine sont plus faibles que celles qui ont été trouvées dans le cas de gestations chez des animaux intacts (tabl. I), si bien que l'excrétion totale d'œstrogènes est plus faible. Il semble donc que les ovaires interviennent en excrétant des œstrogènes. Peut-être modifient-ils les sécrétions totales en jouant sur les formes et les voies d'élimination ? Ces résultats mettent en évidence cependant le rôle important de l'unité foto-placentaire dans la sécrétion des ostrogènes durant la gestation.

De même, bien qu'il y ait d'autres sources de progestérone durant les deux derniers tiers de la gravidité, les ovaires n'étant plus nécessaires au maintien de la ges- 
tation, les corps jaunes à roo jours sont toujours fonctionnels, ce qui tend également à montrer que les ovaires participent aux sécrétions hormonales pendant la gestation chez la Brebis.

TABLEAU I

Excrétion urinaire d'cestrogènes

(les données sont exprimées en $\mu \mathrm{g}$ par $24 \mathrm{~h}$ )

\begin{tabular}{|c|c|c|c|c|c|c|c|c|c|c|c|c|}
\hline \multicolumn{2}{|c|}{ Jours de gestation } & 125 & 132 & 135 & 136 & 137 & $144^{\prime}$ & 145 & 146 & 147 & 148 & 149 \\
\hline \multirow{3}{*}{$\begin{array}{c}\text { CEstradiol } \\
17 \alpha\end{array}$} & Brebis 039 & 28 & 46,1 & 16,8 & 10,5 & 22,2 & 54,3 & 36,2 & 35,3 & 32,1 & 41,5 & \\
\hline & Brebis 835 & & & 82,5 & 31,6 & 39,3 & 40,5 & 59,3 & 28,9 & 53,5 & 78,3 & \\
\hline & $\begin{array}{c}\text { Témoins * } \\
\text { normaux }\end{array}$ & 48,6 & 57,2 & & & 43,9 & & & 76,3 & & 78,6 & \\
\hline \multirow{3}{*}{ Estrone } & Brebis 039 & 10,9 & 14,2 & 16,8 & 10,5 & 22,2 & 14,6 & 7,7 & 8,1 & 8,3 & 16,4 & 38,5 \\
\hline & Brebis 835 & & & 16,5 & 3,9 & 6,9 & 8,8 & 21,2 & 9,2 & 18,8 & 21,3 & \\
\hline & $\begin{array}{l}\text { Témoins * } \\
\text { normaux }\end{array}$ & 28,0 & 32,4 & & & 31,7 & & & 45,6 & & 63,4 & \\
\hline
\end{tabular}

* Moyenne de 4 animaux

En conclusion, il nous faut insister sur le rôle de l'ensemble fœto-placentaire dans la synthèse des œestrogènes durant la gestation. Il serait intéressant de connaître les parts respectives du fotus et du placenta dans cette synthèse, de savoir si, comme pour l'espèce humaine, il y a une synergie entre le placenta et les surrénales et le foie fœtal. AINSWORTh et RYAN (I966) ont d'ailleurs déjà mis en évidence par des expériences in vitro la présence, dans le placenta de Brebis, de certaines enzymes qui interviennent dans la biosynthèse des œstrogènes, une I $7 \alpha$-œestradiol déshydrogénase en particulier.

Reçu pour publication en janvier 1967.

\section{REMERCIFMENTS}

Nous remercions M. P. AufFray, Chargé de Recherches à la Station de Recherches sur l'Élevage des Porcs, qui a bien voulu se charger des interventions chirurgicales. 


\section{SUMMARY}

URINARY ESTROGENS IN THE OVARIECTOMIZED PREGNANT EWE

Investigations on urinary excretion of estrogens by the ovariectomized pregnant Ewe have shown that the main metabolite is $17 \alpha$ estradiol, as it is in normal pregnant ewes. The highest rate was found to be 60 to $80 \mu \mathrm{g}$ per day (fig. I). However, the rate of urinary estrone is lower than that of a normal ewe (table 1 ).

These results point out the importance of the foto-placental unit in the synthesis of estrogens during pregnancy, and also seem to show that the ovaries take part in the secretion, though they are no more necessary to the maintenance of pregnancy.

\section{RÉFÉRENCES BIBLIOGRAPHIQUES}

AINSWORTH L., RYAN K. J., I966. Steroid hormone transformations by endocrine organs from pregnant Mammals. I. Estrogen biosynthesis by mammalian placental preparation in vitro. Endocrinology, $\mathbf{7 9}$, $875^{-883}$.

Denamur R., Martinet J., I955. Effets de l'ovariectomie chez la Brebis pendant la gestation. C. R. Soc. Biol., 149, 2 105-2 107.

Fívre J., Rombauts P., I966. Étude de l'excrétion urinaire des cestrogènes chez la Brebis pendant la gestation. Ann. Biol. anim. Bioch. Biophys., 6, 165-177. 